\title{
LA MANZANA PERdida de RuSSAFA: ESTRATEGIAS DE AUTOGESTIÓN FRENTE A PROCESOS DE GENTRIFICACIÓN
}

\author{
Russafa's Lost Block: Self-management Strategies \\ Versus Gentrification Processes
}

\section{Mijo Miquel Bartual \\ Universidad Politécnica de Valencia, España.}

RESUMEN: En el ámbito urbano se nos plantea abiertamente el reto y la necesidad de desarrollar un nuevo marco que pueda integrar protocolos de participación y gobernanza autogestionados, basados el desarrollo de estrategias bottom-up de resolución de conflictos y transformaciones urbanas. El reto para abordar este nuevo marco reside en redefinir los criterios de salud urbana para establecer nuevas relaciones en el territorio sobre bases más colaborativas, transdisciplinares e inclusivas. Vista la abundancia de solares en la ciudad de Valencia, fruto de la especulación continuada del centro histórico y del primer cinturón de Ensanche, podemos considerarlos como espacios de oportunidad, especialmente en barrios sometidos a procesos de gentrificación acusada. Por ello, hemos lanzado un proyecto de reactivación de una manzana en el barrio de Russafa, esperando mejorar con ello la calidad urbana y generar un espacio en donde sea posible construir comunidad a través de ese mismo proceso deliberativo y transformador.

Palabras Clave: autogestión, gentrificación, salud urbana, espacios de oportunidad, procesos deliberativos, comunidad.

RESUM: En l'àmbit urbà se'ns planteja obertament el repte i la necessitat de desenvolupar un nou marc que puga integrar protocols de participació i governança autogestionats, basats en el desenvolupament d'estratègies d'enfocament ascendent de resolució de conflictes i transformacions urbanes. El repte 
per a abordar aquest nou marc resideix a redefinir els criteris de salut urbana per a establir noves relacions en el territori sobre bases més col-laboratives, transdisciplinars i inclusives. Atesa l'abundància de solars a la ciutat de València, fruit de l'especulació continuada del centre històric i del primer cinturó d'eixample, podem considerar-los com a espais d'oportunitat, especialment en barris sotmesos a processos de gentrificació acusada. Per això, hem llançat un projecte de reactivació d'una illa en el barri de Russafa; esperem millorar amb això la qualitat urbana i generar un espai on siga possible construir comunitat a través d'aquest mateix procés deliberatiu i transformador.

Paraules clau: autogestió, gentrificació, salut urbana, espais d'oportunitat, processos deliberatius, comunitat.

ABSTRACT: In the urban environment we are facing the challenge and the need to develop a new framework that can integrate protocols of participation and governance based on self-management, developing bottom-up conflict resolution strategies and urban transformation. In addressing this new framework, the challenge lies in redefining the criteria for urban health to build new relationships in the area grounded on more collaborative, transdisciplinary and inclusive foundations. The abundance of empty plots in the city of Valencia, the result of continued speculation in the historic centre and the first expansion belt of Eixample, can be considered as opportunity spaces, especially in the neighbourhoods undergoing gentrification. As a result, we have launched a project to reactivate a block in the neighbourhood of Russafa, hoping thereby to improve urban quality and create a space where a community can be built through the deliberative and transformative process itself.

KEYWORDS: self-management, gentrification, urban health, oportunity spaces, deliberative processes, community. 


\section{Introducción}

$\mathrm{C}$ uando analizamos en la actualidad la evolución de las ciudades, vemos cómo el urbanismo se plantea no tanto la expansión de las ciudades más bien sometidas a procesos de decrecimiento, como el reajuste de zonas ya urbanizadas, especialmente aquéllas que forman parte del centro histórico, sometidas a fuertes procesos de gentrificación, aún parcialmente paralizados por la crisis. Los modelos de planificación urbana empleados durante todos los años de desarrollismo, de carácter formal y reactivo, basados en la idea de proyecto estable (los Planes Generales de Ordenación Urbana), están claramente obsoletos tanto por su dirección general orientada al crecimiento constante como por las prioridades establecidas que suelen situar al tránsito como su primer bastión, por no hablar de las oportunidades desaprovechadas de gestión lábil que abren las tecnologías interactivas.

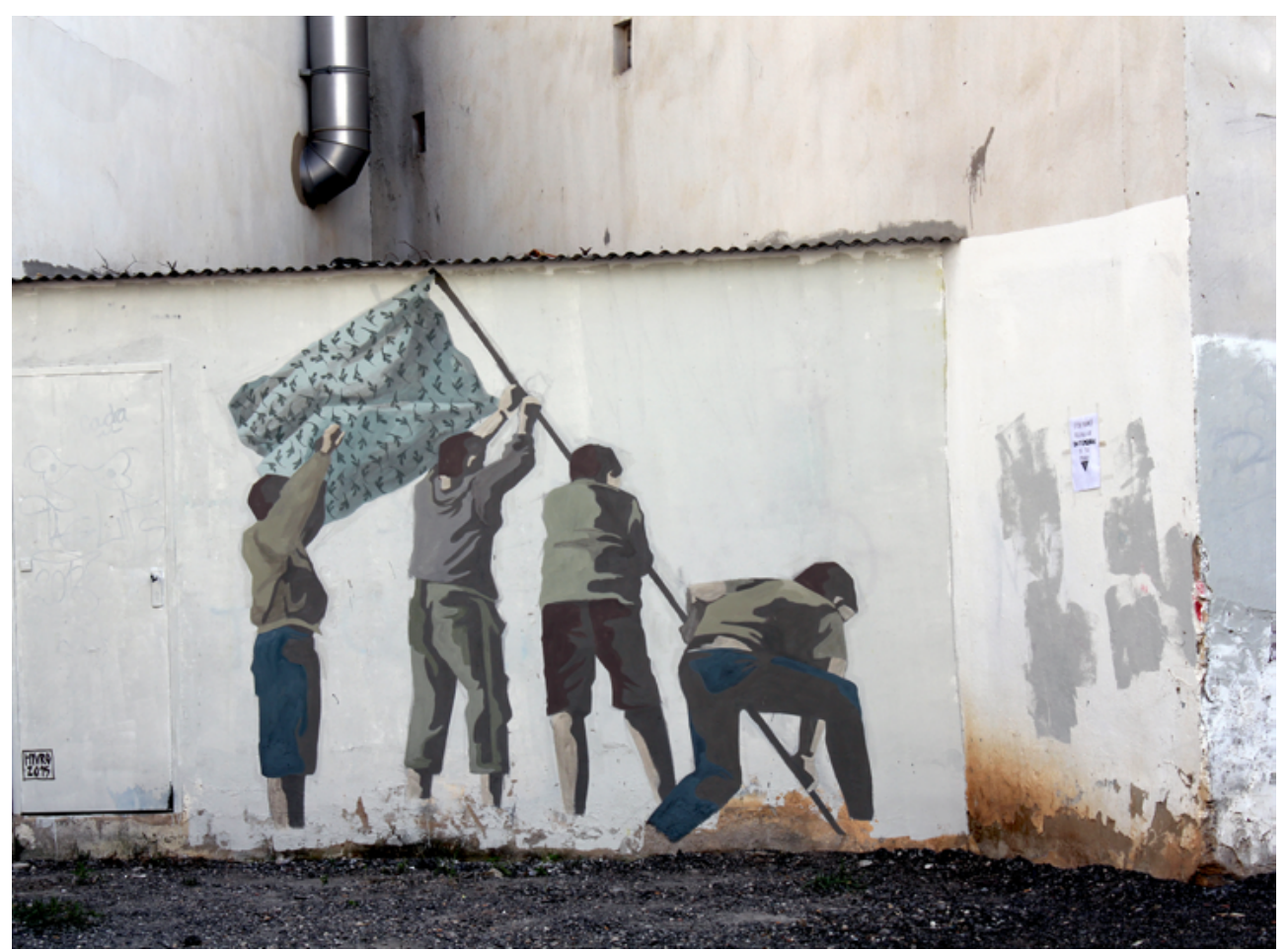

Figura 1 .

De la misma manera, la metodología de trabajo ha privilegiado históricamente la figura del experto frente al conocimiento del territorio aportado por el ciudadano y a menudo ha excluido, aún en los últimos tiempos bajo las 
premisas de una teórica participación, a gran parte de las asociaciones de vecinos y plataformas que se oponen a una planificación basada en criterios económicos y especulativos, así como a ciudadanos que a título personal, proponen participar en la toma de decisiones a través de las Juntas de Distrito. Si bien en algunas ciudades del territorio nacional están corriendo nuevos aires y estas metodologías vuelven a estar dotadas de sentido en tanto mecanismos que antaño se impusieron como garantes de una porosidad democrática, aún son rara avis y están lejos de estabilizarse. Esperemos que las transformaciones vayan más allá de un cambio de manos de los grupos de influencia y se consiga un funcionamiento equilibrado que integre una multiplicidad de interlocutores y de puntos de vista, incluyendo no sólo a los expertos sino también a los ciudadanos en su sentido más amplio, es decir, que no sean exclusivamente varones, de raza blanca, en edad productiva y con necesidades de tránsito duro, prolongando a nuestra manera la ceguera poblacional que reinaba en el ágora griega y que invisibilizaba a más de la mitad de su población. Por tanto, en el ámbito urbano se nos plantea abiertamente el reto y la necesidad de desarrollar un nuevo marco que pueda integrar protocolos de participación y gobernanza autogestionados, basados en el desarrollo de estrategias bottom-up de resolución de conflictos y transformaciones urbanas. El reto para abordar este nuevo marco reside en redefinir los criterios de $s a$ lud urbana para establecer nuevas relaciones en el territorio sobre bases más colaborativas, transdisciplinares e inclusivas, que incluyan el punto de vista de disciplinas recientemente orientadas a procesos participativos y hacerlo en un rango de acción que vaya más allá del ciudadano tipo para incluir cuestiones como el género, la edad, la nacionalidad o los parámetros de movilidad elegidos.

Esta deriva dentro del urbanismo sucede de forma paralela a la que experimentan numerosas disciplinas que son progresivamente conscientes de las necesidades de aplicar un enfoque transdisciplinar al análisis de situaciones así como a conceptos generales que se habían desarrollado de forma progresivamente sectorial, conceptos tan tradicionalmente acotados a una ciencia concreta como el de salud. No obstante, en la definición que hoy en día podemos hacer de esta noción, tal y como se muestra en el diagrama, resulta evidente que su complejidad exige un planteamiento multifactorial que rede- 
fina políticas públicas orientándolas a este fin. La primera Conferencia Internacional sobre la Promoción de la Salud reunida en Ottawa ya en 1986 emite una carta dirigida a la consecución del objetivo «Salud para Todos en el año 2000» en respuesta a la creciente demanda de una nueva concepción de la salud pública en el mundo. La Carta de Otawa ha incidido especialmente en la importancia de la capacidad de las personas y de las comunidades para controlar los determinantes de su salud y poder mejorarla. Esto implica poder actuar no únicamente a nivel corporal sino también en el arco superior del esquema previo, en cuanto a las condiciones socioeconómicas, ambientales y culturales que tienen un impacto directo en el estilo de vida individual. La Carta pone el énfasis en los factores protectores y preventivos, incide en considerar que la participación y la acción social son esenciales así como en el valor de las políticas públicas referentes al entorno en sí. A nivel institucional, este cambio de enfoque está incluido en la mayoría de agendas, pero a menudo no hay un correlato coherente en metodologías y prácticas.

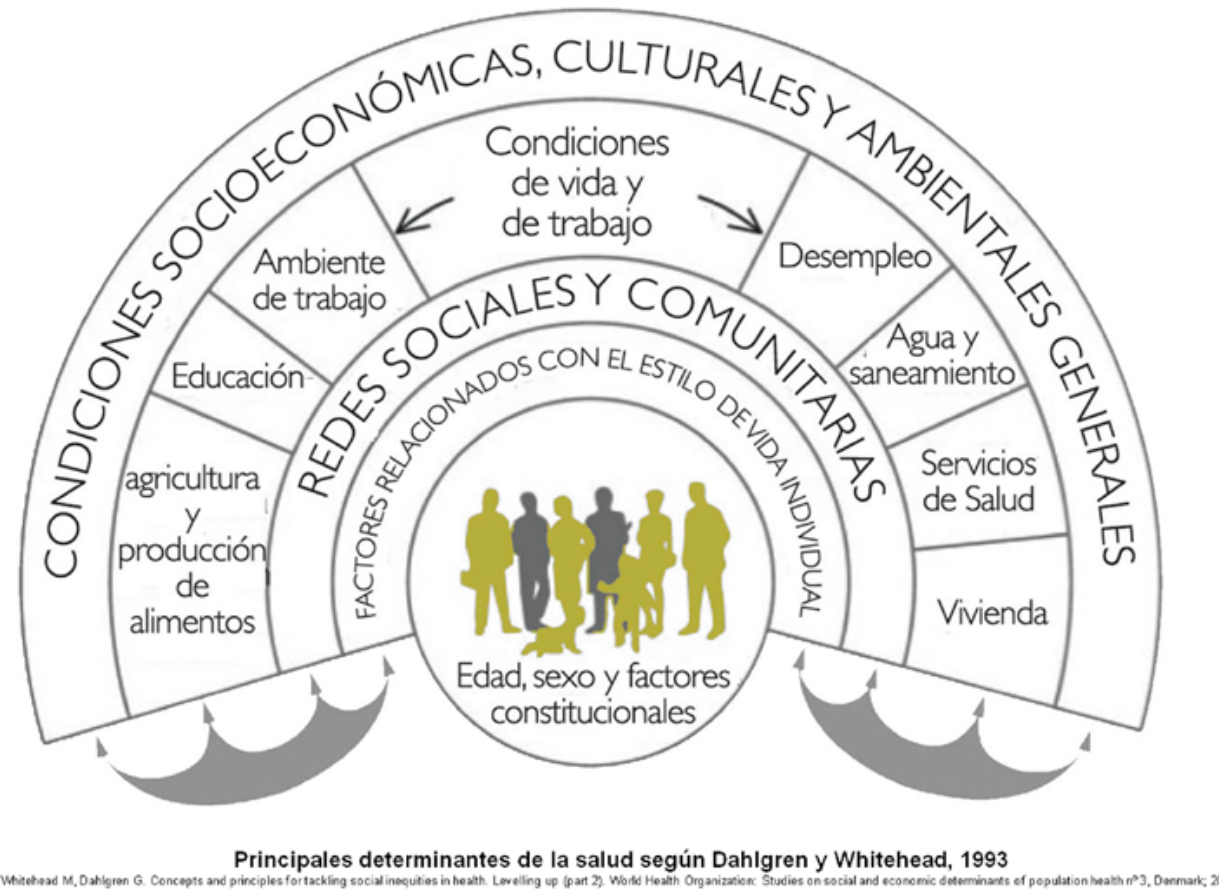

Figura 2. Whitehead M, Dahlgren G. Concepts and Principles for Tackling Social Inequities in Health. Levelling up (part 2) World Health Organization: Studies on Social and Eco-

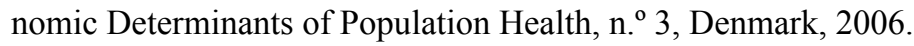


A raíz de esta apertura hacia nociones ecológicas, sociológicas y participativas, el tratamiento de la ciudad ha cobrado una importancia relevante, no sólo porque la mayoría de la población vive en ellas sino porque el ciudadano empieza a dibujarse como un posible sujeto revolucionario equivalente a la fuerza que el proletariado tuvo en su momento. Con un $60 \%$ de la población viviendo en urbes, no podía ser menos, pero la condición ciudadana va más allá del hecho de vivir en un entorno urbano y hace referencia a la construcción de un sujeto metropolitano sometido a las tensiones propias del postfordismo. Somos habitantes de las metrópolis y como tales, podemos construir una identidad colectiva y articulada desde donde transformar no sólo nuestro entorno inmediato, sino los parámetros por los que se rige el pacto de realidad en respuesta a una precarización generalizada de derechos, afectos y relatos que ha contribuido a la disolución de esta identidad, no necesariamente entendida bajo la idea de fortaleza sino más bien, como una constelación de referencias, como una «biografía en constante traducción». ${ }^{1}$ En estas coordenadas, cualquier análisis urbano debe ir contrastado con un mapa de agentes sociales y relaciones para entender verdaderamente el potencial que nos abre nuestra condición ciudadana en tanto co-autores de nuestros espacios.

Vista la abundancia de solares en la ciudad de Valencia (espacios al aire libre que nuestro clima mediterráneo permite utilizar casi todo el año), fruto de la especulación continuada del centro histórico y del primer cinturón de Ensanche, podemos considerarlos como algo más que una cicatriz de esta década de maltrato urbano y celebrar su existencia en tanto espacios de oportunidad: lugares donde aislarse y descansar, donde realizar encuentros y celebraciones, practicar juegos y deportes, habilitarlos como aulas, talleres, auditorios o escenarios, diseñar jardines, cultivar para el autoconsumo, instalar fuentes de energía renovable, renaturalizar la ciudad con huertos urbanos, jardines, crear biodiversidad, reducir la contaminación y combatir las emisiones de $\mathrm{CO} 2$. Territorios en donde podemos proyectarnos y a través de esta proyección, renovar los vínculos en tanto comunidades que se construyen de forma compartida en el hacer y en el pensar, siendo la deliberación y sus emociones las que nos permiten construir un «nosotros» que muchos otros

1. Beck, Ulrich y Beck-Gernsheim, Elisabeth (2002): Individualization. Londres: Sage. 
prefieren al término comunidad, sometido a la sospecha de la inmunidad, de la construcción excluyente de un territorio basada en la protección frente a los otros.

Teniendo en cuenta que los territorios compartidos por el arte y la arquitectura son cada vez más extensos, tanto en lo que respecta a la sensibilidad respecto a temáticas como al uso de herramientas de participación, vamos a comentar un proyecto en proceso en el que hemos trabajado aplicando un análisis multifactorial a nuestro propio entorno y hemos actuado en consecuencia, ampliando la propuesta a la ciudadanía en un barrio en el que los procesos de articulación interna y los deseos de autogestión del territorio son cada vez más sólidos y conscientes, Russafa. Si tomamos la ciudad como un laboratorio de experimentación en cuanto a nuevas formas de gobernarse, deberíamos estar atentas a los procesos emergentes que rompen la lógica de las exigencias dotacionales y pasan a solicitar la autogestión de su territorio en pleno ejercicio de democracia contributiva, porque abren espacios de oportunidad prototipables que pueden servir para multiplicar iniciativas, promoviéndolas también desde las instituciones.

\section{El marco barrial}

El barrio de Russafa es fruto de la fusión del núcleo histórico del pueblo de Russafa y la primera ampliación programada de Valencia, realizada entre finales del siglo XIX y principios de Xx, y denominada el Ensanche. Guarda un parentesco claro con las intervenciones urbanísticas realizadas en Barcelona bajo las órdenes de Ildefonso Cerdá, que proyectará la ampliación de la ciudad realizando un ordenamiento por manzanas con arcadas en cuyo interior habría jardines en principio planificados como públicos. Cerdá intentaba con ello democratizar la ciudad, ofreciendo similares condiciones de vida (horas de sol, vistas, espacios verdes intercalados) a todos los ciudadanos, estableciendo un vínculo claro entre salud individual y condiciones urbanísticas. No obstante, su plan fue llevado a cabo de forma diferente a la proyectada ya que el acceso a las zonas internas se convirtió inmediatamente en privado, reduciéndose además sus dimensiones y eliminando en parte los espacios verdes. 


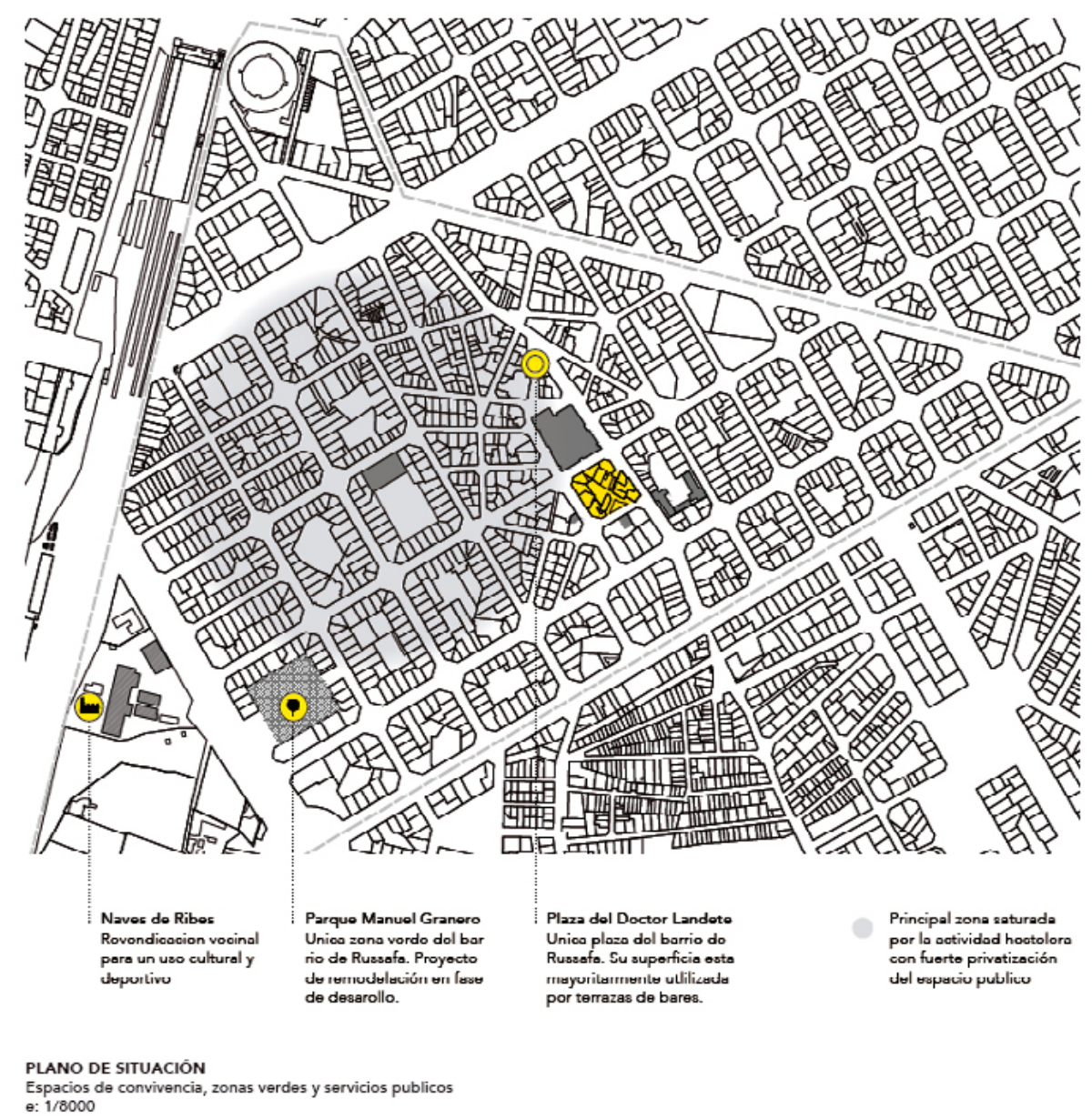

Figura 3. Plano realizado por Estelle Jullian para el proyecto de La Manzana Perdida Emergents 2015.

En el caso del Ensanche valenciano, la burguesía floreciente se instala en estas nuevas zonas, especialmente en la demarcada por la avenida Antiguo Reino de Valencia, y el río Turia, convertido en una amplia zona ajardinada tras realizarse su desviación definitiva, aunque Russafa estuvo más bien destinada a las clases medias bajas de menores rentas. En la actualidad, este barrio cuenta con 23779 habitantes y está delimitado por la avenida de Peris y Valero, la avenida del Antiguo Reino de Valencia y el eje que conforman las calles de Alicante, Gibraltar y Filipinas. En él, está prevista desde hace más de dos décadas la realización de un gran Parque Central en el suelo liberado 
por el soterramiento de las vías del tren y la reunificación de esta parte de la ciudad, separada históricamente por el trazado ferroviario.

Esta última cuestión nos da la clave de la transformación que ha sufrido el barrio en los últimos 20 años. En torno a su espejismo, se han producido numerosas operaciones especulativas e intentos de cooptación por parte de promotoras y constructoras del enriquecimiento que supondría su realización tanto en términos de valor catastral como de valor inmobiliario. Resumiremos brevemente el proceso que, por ser un clásico, no deja mucho campo a la duda. Russafa fue durante muchos años un barrio popular, en donde abundaba el pequeño comercio local que abastecía a sus habitantes, un barrio con sus centros de reunión y desarrollo de la vida social, dos iglesias, un mercado, un parque, dos colegios públicos y una tradición fallera muy arraigada que articulaba su vida social en torno a los casales.

A pesar de ser colindante con el centro histórico de la ciudad, se consideró que el distrito de Russafa era periférico en su composición. Atendiendo a la evolución del barrio constatada por Francisco Torres, ${ }^{2}$ sociólogo especializado en el estudio de las dinámicas de convivencia en barrios multiculturales, veremos cómo tras un período incluso de perdida de población, empiezan a llegar a partir de los 90 nuevos vecinos autóctonos, jóvenes estudiantes y profesionales en su mayoría, y los vecinos extranjeros, que en enero de 2005 constituían el 15,9\% del total del vecindario. En esta primera fase, la población extranjera que se integra en el barrio es fundamentalmente magrebí y lo hace tanto instalándose a vivir en él como abriendo simultáneamente comercios halal, teterías y otras tiendas que abastecen a la población magrebí no sólo del barrio sino de otras zonas. De esta manera, Russafa se convierte en un espacio de sociabilidad y no sólo de alojamiento para dichos inmigrantes. Este esquema se reproduce en menor grado en lo que respecta a la población subsahariana y a la china, son transformaciones lentas en las que las familias integran a los niños mediante los establecimientos educativos y ellas mismas lo hacen a través de los comercios de proximidad que abren en sus mismas

2. Torres, F., Las dinámicas de la convivencia en un barrio multicultural. El caso de Russafa (Valencia), en Papeles del CEIC, vol. 2006/1, papel n ${ }^{\circ} 23$, CEIC (Centro de Estudios sobre la Identidad Colectiva), Universidad del País Vasco, en http://www.ehu. es/ CEIC /papeles/23.pdf. 
calles. De la misma manera, se formalizan las primeras asociaciones de inmigrantes que fomentan una integración a todos los niveles mediante asesoramiento jurídico, clases de español, clases de árabe, etc.

Esta primera ola migrante, asumida sin gran problema por un barrio, contrasta con la manera en que se produce la segunda ola. Inicialmente podríamos hablar de una situación de co-presencia residencial, distinta de la experiencia anglosajona del barrio étnico y de la inserción residencial segregada, es decir, se comparten los espacios públicos a causa de las residencias mixtas pero se separan los espacios íntimos, produciéndose lo que viene a llamarse inserción urbana, tal y como especifica Torres. ${ }^{3}$ En el caso de Russafa, podemos hablar de un primer momento de co-presencia residencial previo al paulatino proceso de inserción integrada, quebrado a su vez por las dinámicas propias de la gentrificación que demonizan al migrante para expulsarlo en una segunda fase.

El hecho de que en un primer tiempo se elija este barrio como destino de migrantes y artistas, responde al mismo esquema que se ha reproducido en muchas otras ciudades europeas de determinado tamaño, en las que un cierto cosmopolitismo unido a la degradación de zonas del centro histórico o adyacentes permite la concentración en ellas de esta mezcla de componentes poblacionales. La mayoría de la población original censada a principios de los 90 compró sus viviendas en el momento de su construcción por lo que la población respondía en ese momento a dos características diferenciales respecto a otros barrios: su origen era fundamentalmente valenciano, con poca migración nacional, y su media de edad era bastante elevada. Esto significa que en el momento en que empieza a realizarse el relevo generacional, a partir de la década de los 90, la existencia de este parque inmobiliario envejecido va a favorecer la entrada de inquilinos de bajo poder adquisitivo. Esto hace que, en pleno boom inmobiliario generalizado, en la zona se produzca un fenómeno de doble nivel que ya Torres achaca a procesos de especulación y que se ve recalcado por Moncusí ${ }^{4}$ en estudios posteriores. Por un lado, se

3. Torres, F., íbid.

4. Moncusí Ferré, Albert, Nuevos y viejos vecinos en dos barrios de Valencia (Orriols y Russafa), Departament de Sociologia i Antropologia Social, Ajuntament de València, Àrea de Progrés Humà. Regidoria de Benestar Social i Integració, València, 2009. 
realiza la promoción de edificios de nueva planta. Por otro, se produce un deterioro de las zonas en las que se alojan inmigrantes cuyas casas son ya de menor calidad. Algunas de esas casas, abandonadas, sufren fenómenos de ocupación simultáneos a la introducción de la compra-venta de drogas en esos sectores, situación ante la que, voluntaria o inadvertidamente, la policía hace reiteradamente la vista gorda. En resumen, tras una primera década de transformación progresiva de la población, a partir del 2000, determinadas zonas (curiosamente, las limítrofes al proyecto del Parque Central se cuentan entre ellas) empiezan a degradarse sensiblemente por la dejación de control policial, el deterioro de casas y su ocupación ilegal, la aparición y multiplicación de solares en las zonas en las que ya sólo se puede contemplar el derribo.

Paralelamente, se lanza la idea de que el barrio está invadido de inmigrantes, que su proporción es mucho mayor que en otras zonas, a pesar de que las estadísticas demuestran lo contrario, ya que se ha producido un estancamiento e incluso decrecimiento de la población extranjera a lo largo del 2000. Como bien sabemos, la apariencia de concentración étnica es un fenómeno construido también de forma subjetiva, no importa tanto la presencia real de población de determinado origen étnico como la creencia en la misma. La primera visión de riqueza multicultural que se tuvo al principio se ve sustituida por una de «invasión», fomentada por grupos de ultraderecha, así como por determinados medios de comunicación y otros agentes de identidades e intereses no declarados. A consecuencias de todo ello, se producen disensos de importancia dentro de las asociaciones por lo que algunos de sus miembros así como parte de la población española renovada, en su mayoría profesionales liberales, artistas, etc., entienden que hay que iniciar una nueva plataforma en la que también se integre a la población extranjera y que sirva para convertirse en interlocutora válida de cara a las administraciones: de esta manera se funda en octubre de 2002 la Plataforma per Russafa, ${ }^{5}$ que jugará un papel importante en la definición del barrio durante la última década, agrupando a diferentes entidades vecinales y coexistiendo con la asociación de vecinos inicial.

5. www.russafa.org. 
Tras esta construcción de la imagen de deterioro barrial con su consecuente reducción asociada de precios de venta y aprobación de derribos de edificios en mal estado, posterior a su ocupación ilegal pero consentida, se sucede la promoción y construcción de nuevas viviendas de cierta calidad, para sentar las bases de un proceso de gentrificación. Afortunadamente, el barrio cuenta con un denso tejido asociativo que inicia, desde la mencionada Plataforma de Russafa, a la que se suman otras asociaciones, el largo período aún no concluido de manifestaciones vecinales, actos reivindicativos, recogida de firmas y participaciones en los Plenos de la Junta Municipal con acciones legales orientadas fundamentalmente a dos cuestiones: bloquear la construcción del parking en la única zona verde existente y activar la construcción de un nuevo colegio en la calle Puerto Rico para paliar la carencia de plazas escolares, objetivos ambos alcanzados con éxito.

No obstante a partir de 2004, se pone en marcha el programa policial MAS con la intención de higienizar el barrio intensificando la presencia policial en las calles, el control de sin papeles, el derribo deseado de casas tomadas, una limpieza de cara a toda prisa y con toda violencia. Desde las altas instancias, se ha percibido la oportunidad económica que supone el barrio, se ha sembrado miseria y se quiere recoger dinero: se inicia en la zona el espejismo inmobiliario declarado. De la multiplicación de locutorios, se pasa a la profusión de inmobiliarias, los bancos de la esquina conceden créditos como si fueran bizcochos, las tiendas de barrio suspiran y sueñan un destino burgués, los primeros carteles de lofts de lujo aparecen en el horizonte.

Simultáneamente, se siguen reivindicando equipamientos básicos que no se ejecutan ya que, según nos aseguran, cuando la promesa del Parque Central se convierta en una realidad en un futuro luminoso, todas nuestras necesidades serán cubiertas con creces. En la actualidad, han comenzado finalmente las obras correspondientes a la primera fase del parque y está en el aire la adjudicación y usos de las Naves de Ribes, en las que la Plataforma exige la implementación del polideportivo y centro cultural que los vecinos vienen reclamando desde hace siglos así como su autogestión. Estos años de dinámicas antagonistas concretas, basadas no obstante en la concertación, en colaboraciones y desacuerdos, han tenido consecuencias no menospreciables para el tejido asociativo: se ha consolidado una red de relaciones y contac- 
tos de personas activas de los diferentes sectores del barrio que ha llevado a la constitución de una Asamblea Consultiva en la que participan más de 15 asociaciones diferentes, erigiéndose en interlocutora reconocida por todas las partes implicadas, incluido el propio Ayuntamiento.

Como vemos, resulta fundamental para la «salud» de la ciudad, que se fomente activamente la existencia de un tejido asociativo en todos los barrios que permita mediar entre lo que la Administración considera beneficioso y la realidad de la población que lo vive y que, por tanto, está en una posición inmejorable para captar las necesidades, adaptaciones y proyectos viables en cada zona. Desde la posición institucional, no sólo no se debería entrar en una lógica antagonista con los propios ciudadanos y sus asociaciones, como viene siendo reiteradamente el caso en esta ciudad, sino que se debería fomentar la creación y funcionamiento de plataformas de mediación Ayuntamiento-sociedad civil de manera que la co-gestión participativa fuera una realidad integrada y no una excepción ganada a pulso.

\section{Problemáticas actuales}

Dentro de las problemáticas generales del barrio, debemos citar en primer lugar la culminación del proceso acusado de gentrificación que ha provocado la exclusión de colectivos poblacionales, la paulatina desaparición de comercios de proximidad, la privatización del espacio público y un grave problema de saturación hostelera con las consecuencias que ello implica: deterioro de la calidad de vida, incremento de los niveles de ruido, multiplicación de las necesidades de aparcamiento en horario de ocio, etc. Todo ello, tal y como se detalla en la memoria general presentada por la Plataforma de Russafa sobre la reivindicación de las Naves de Ribes, no hace más que incrementar las necesidades y carencias del barrio en cuanto a infraestructuras públicas deportivas y culturales, espacios verdes, espacios de relación, etc. deteriorando aún más la calidad de vida del ciudadano en general y, especialmente, de los colectivos más débiles como pueden ser la tercera edad, los migrantes o los niños.

Así, el «Informe participativo sobre necesidades del barrio de Russafa» realizado por la Plataforma con la colaboración del estudio de sociólogos $\mathrm{La}$ 
Dula $^{6}$ incide en la valoración de la cultura y el deporte como «herramientas para la cohesión del vecindario, como potenciadoras de las formas de sociabilidad y relaciones sociales del lugar». La conclusión que emerge de este informe en lo referente a carencias diagnosticadas por los vecinos evidencia la «necesidad de espacios para la cohesión social y vertebración del barrio, haciendo hincapié en que sean lugares para el encuentro de grupos sociales diversos». En dicho informe se indica también que ha sido la alta densidad de movimientos asociativos en el barrio de Russafa la que ha frenado las consecuencias negativas del proceso acusado de gentrificación y sus efectos colaterales.

Por ello, consideramos necesario seguir contando en cualquier proceso con la participación del máximo número de colectivos y personas de manera que se pueda favorecer la cohesión social del barrio generando un mejor desarrollo, así como mayor alcance e inclusividad de las actividades sociales, culturales, de ocio y deportivas, que se puedan proponer. Igualmente, consideramos de la mayor importancia generar espacios de deliberación y articulación interna de las comunidades, que a su vez puedan funcionar posteriormente como interlocutoras activas de las instituciones, proponiendo y estableciendo marcos a los procesos participativos propuestos desde arriba. Dichos espacios no se producen únicamente a nivel conceptual sino que tienen un correlato físico y de ello se trata este artículo, de cómo localizar y dinamizar espacios de oportunidad en los que se potencien ambas facetas, la adecuación física y la proyección conceptual, como estrategia posible de oposición a la gentrificación y vaciamiento de un barrio.

\section{La manzana de la discordia}

Hace ya unas décadas que en pleno centro de Russafa se esconde una joya, un despropósito, una oportunidad única, un foco de suciedad, un espacio verde maravilloso... La manzana dibujada por el encuentro de las calles Reina Doña María, Barón de Cortés, Mestre Aguilar y Poeta Al-Russafi, entre instalaciones tan frecuentadas como son el Mercado de Russafa y la Nueva Biblioteca Al-Russafi, junto al colegio Balmes, es actualmente un descam-

\section{6. http://ladulaparticipacio.com/la-dula-participacion/.}


pado en el que se aparcan coches, se acumula la basura y cuyos solares sirven de nido de ratas. Un espacio insalubre y sin uso que nadie quiere mirar. El Plan General de Ordenación Urbana (PGOU) de 1988 prevé para esta zona una edificación tipo manzana similar a todas las demás trazadas con geométrica precisión. No obstante, tras más de 22 años desde su aprobación, el espacio se ha mantenido a caballo entre dos proyectos: el trazado anterior con fincas antiguas parcialmente habitadas que se encuentran fuera de ordenación, que no siguen el trazado rectangular propio de las manzanas del Ensanche, junto con fincas de nueva planta y un espacio interior donde conviven fragmentos de aceras que las nuevas fincas han debido construir y el barro.

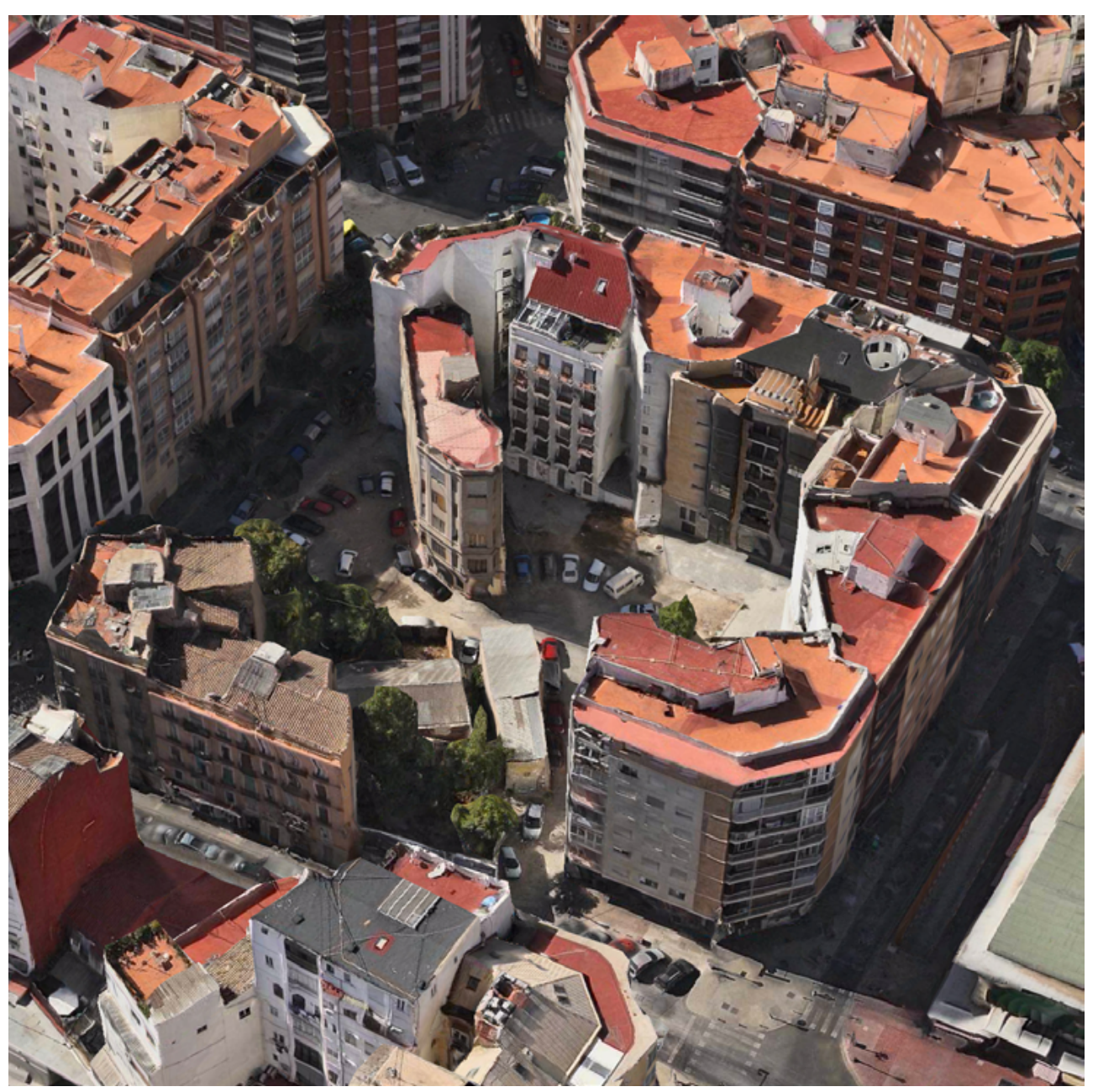

Figura 4. Vista aérea de la Manzana Perdida. 
En noviembre de 2004 se adjudicó a Inmobiliaria Urbis SL un PAI que delimitaba esta zona a través de una unidad de ejecución y el correspondiente Plan de Reforma Interior, pero la Concejalía de Territorio, a la vista de las numerosas sentencias que han recaído sobre el Ayuntamiento de Valencia por incumplir la LRAU y el Reglamento de Planeamiento en cuanto a ausencia de transparencia en la tramitación, exigió nuevamente la exposición pública para evitar una nueva condena. En 2005 se produjo una cesión de la condición de agente urbanizador de Inmobiliaria Urbis a Valencia Constitución SL. En 2006 se expuso nuevamente la documentación al público y años después el PRI quedó obsoleto. Mientras tanto, son los vecinos de Russafa los que padecen esta situación, fruto de un cúmulo de irregularidades, retrasos y desinterés por parte de la Administración que gestó el Plan.

En este estado de cosas, propusimos mirar de otra manera este espacio para descubrir el potencial que alberga y pusimos en marcha un proceso de reapropiación que transformara el espacio entendiendo sus carencias como oportunidades. Para ello, lanzamos la convocatoria de una primera jornada de visibilización del espacio el 26 de junio de 2015, en la que, tras una primera fase de propuesta e implicación de diferentes colectivos, participaron finalmente los siguientes: la Plataforma de Russafa, la Asociación de Vecinos de Russafa-Gran Vía, el grupo de consumo de Russafa, el AMPA del Colegio Balmes y los propios vecinos que rodean la manzana. El desconocimiento que muchos de ellos manifestaron sobre la propia manzana en sí, su emplazamiento y estado, nos reafirmó en nuestro propósito ya que evidenció hasta qué punto somos capaces de generar puntos ciegos en nuestra percepción del territorio que habitamos cotidianamente.

Planteamos la realización de intervenciones artísticas conscientes de la capacidad que tienen de visibilizar un espacio permitiendo simultáneamente la transformación de su aspecto exterior, conquistando visualmente un territorio. Para ello, contamos con las intervenciones de Hyuro, Anaïs Florin, Vira Lata, Mijo Mik así como de algunos alumnos del Máster Producción Artística (Joana Mollá, Chao Yang y otros performers invitados que planteaban procesos de visibilización relacional) seleccionados mediante la convocatoria 
«Solares Lunares» dentro de SELECTA 2015.7 Simultáneamente, el equipo de Carpe Vía ${ }^{8}$ realizó su tercera edición del Viaje al Interior de la Noche, se equipó con su Maleta de los Viajes, aterrizó en el descampado y preparó una exposición interactiva para que los viandantes pensaran qué les gustaría tener en un lugar tan especial como La Manzana Perdida. Obtuvieron resultados previsibles en cuanto al diagnóstico negativo del espacio: coches abandonados, edificio en ruinas que estorba en medio de la manzana, los solares como focos de suciedad, etc.; y otros no tanto en cuanto a los aspectos positivos: plaza con pavimento adoquinado en uno de los lados, forma irregular de la manzana para un parque infantil diferente, es una de las pocas zonas de Russafa con suelo de tierra. En cuanto a las propuestas de actuación sobre el espacio, fueron las siguientes: un pequeño estanque limpio, zona verde, parque infantil y de mayores, bar con terraza, cine de verano a la fresca, más iluminación, un hospital de barrio, sembrar árboles, plantas, césped, tirar el edificio en ruinas para que haya más espacio, un huerto para niños y mayores... Más allá de las ideas escritas, muchos vecinos se animaron a dibujar sus propios diseños sobre el plano y diferentes alumnos de arquitectura realizaron pequeños proyectos de transformación del espacio que también expusieron en los muros de los solares.

A lo largo del día, también llevamos a cabo juegos para niños, un primer sondeo sobre el posible futuro de la manzana, una cena de sobaquillo, y algunas actividades más, generando un espacio de experiencias compartido en el que convivieron diferentes generaciones, y dimos a conocer el espacio y su situación. En resumen, tras la construcción de una narración compartida a través del análisis inicial del espacio, llevamos a cabo un proceso de visibilización de carencias seguido de otro de visualización de deseos y de un tercero de transformación vivencial del espacio y, por tanto, de las relaciones, emociones y experiencias asociadas a él así como del discurso posibilitador utilizado para comunicarlo.

\footnotetext{
7. http://selecta.blogs.upv.es/selecta-2015/.

8. http://www.carpevia.org/portfolio/viaje-a-ruzafa/.
} 
Tras el éxito de esta jornada, llevamos a cabo una investigación más completa junto con Estelle Jullian ${ }^{9}$ y Paula Roselló, ${ }^{10}$ sobre las circunstancias que rodean y determinan este espacio, advirtiendo una situación administrativa compleja dentro de la normativa vigente, sometida a un Plan General de Ordenación Urbanística PEP-2 que bloquea el espacio a la espera de una promotora que urbanice la zona, deja fuera de plan algunas viviendas y no ejecuta los viales marcados ni el espacio verde central, permitiendo no obstante la ocupación diaria por parte de vehículos privados. A pesar de ello, consideramos que la manzana es un espacio de oportunidad en muchos ámbitos ya que toca a la memoria histórica puesto que es el único rastro de la trama antigua del barrio y aún posee restos parciales de la calzada de piedra del Ródeno que se remonta a cuando Russafa aún era un pueblo. Igualmente, supone el mantenimiento de una variedad urbanística, no sólo en términos de trama y disposición, ya que esta manzana con un trazado diagonal irregular y una pequeña plaza en la retícula del Ensanche, supone un grado de diversidad mayor y por tanto de riqueza espacial. De la misma manera, en términos de ecología, la necesidad endémica de esponjamiento de una trama rural posteriormente urbanizada muy compacta se vería compensada por la creación y mantenimiento de dos puntos verdes: el espacio de encuentro que cuenta ya con árboles de varios metros y un posible huerto pedagógico. Por último y no menos importante, la inclusión social; este factor se desarrolla a diferentes niveles ya que supone la creación de un espacio inclusivo no determinado por el consumo, donde desarrollar actividades intergeneracionales, abriendo espacios para los que no suelen tenerlo: inmigrantes, niños, mayores...

Por todo ello, consideramos que tanto en términos ecológicos como sociales y urbanísticos, resultaría pertinente su activación en diferentes fases y con diferentes grados de implantación: desde una cesión de usos temporal hasta la transformación definitiva. Por otra parte, el planteamiento de un proceso participativo previo serviría de proceso de mediación entre las diferentes posturas que ya se han venido recogiendo, posiciones generalmente complementarias pero también contrapuestas: la necesidad de espacios de

\footnotetext{
9. http://atelierinterlope.com/.

10. http://encajesurbanos.com/.
} 
aparcamiento así como de espacio para perros, por ejemplo, ambas actividades difícilmente compatibles con espacios de relación y de juego.

Aprovechando la convocatoria Emergents ${ }^{11}$ de la Universidad de Valencia para proyectos de creatividad, innovación e inclusión social, presentamos un proyecto en el que se proponía una segunda jornada de convivencia en la manzana que permitiera visibilizar aún más este espacio y mostrarlo como una ventana de oportunidades para el barrio, implicando de nuevo a los vecinos en un proyecto de transformación del mismo, así como la preparación de un dossier técnico como punto de partida para la puesta en marcha de un proceso de participación que reflexionara sobre la manzana y permitiera desbloquearla propiciando mejoras para todo el barrio. En esta convocatoria nos han concedido un premio de accésit, por lo que en la actualidad nos encontramos en proceso de diálogo con el Ayuntamiento para activar el plan de actuación barrial según lo hemos especificado.

\section{Conclusiones}

En relación a estas temáticas, nos parece importante recalcar la idea de la ciudadanía inteligente frente a la mediatizada implementación de las Smart Cities en la construcción de un posible futuro ciudadano. Estamos plenamente convencidas de que la tecnologización de las ciudades entendidas como datos beneficia fundamentalmente a las grandes empresas que la implementan y tan sólo tangencialmente al ciudadano medio que en muy poco recuperará la capacidad de decisión y propuesta. El planteamiento centralizado de los datos y su gestión opaca no nos permiten albergar grandes esperanzas aún a sabiendas de lo que estos sistemas permitirían si fueran acompañados de un cambio de actitud, ya que posibilitarían una flexibilización y horizontalidad en la gestión de la ciudad. Proponemos, como tantos otros están reivindicando en la actualidad, la emergencia de una ciudadanía inteligente y para conseguirlo, habría que modificar tanto el enfoque general sobre las maneras de proyectar ciudades autosuficientes, como la necesidad de llevar a cabo para conseguirlo una serie de inversiones mínimas, inversiones que nadie pone en duda cuando

11. www.emergents.info. 
se alega la gestión eficaz de la ciudad en cuestiones como los aparcamientos, la calidad del aire, o tantos otros parámetros registrados y movilizados por las tecnologías urbanas que priorizan una gestión neutra, ajena, externalizada y probablemente mercantilizable, pero que sí se ponen en duda cuando se trata de fomentar dinámicas de autogestión que permitan la puesta en acto de la idea de democracia contributiva. Por otra parte, la construcción de redes de personas nos parece la única posibilidad viable a la hora de poder constituir una alternativa de autogestión, objetivo que también se ve favorecido por el desarrollo de las nuevas tecnologías, sí, pero no únicamente, por lo que deberían entenderse como complemento estructural y nunca como fin último.

Por todo ello, consideramos que poner en marcha proyectos de reactivación de solares en un barrio determinado constituye una acción que promueve la salud a diferentes niveles: social, ecológico, corporal e incluso económico. De manera escueta, se pueden resumir sus beneficios en la capacidad de generar red social, establecer vínculos entre vecinos, recuperar espacios indefinidos sobre los que proyectar necesidades comunes, esponjar tramas urbanas con espacios de reunión no planificados, empoderar la ciudadanía mediante la recuperación de espacios de autogestión, mejorar el medio ambiente mediante la reforestación urbana, etc. De forma más detallada podríamos decir que frente a una lógica de gobierno de las ciudades basada en las restricciones y en las prohibiciones, como viene siendo la tónica habitual de las normativas que rigen las ciudades españolas, se podría trabajar en el fomento de las buenas prácticas: adecuación de espacios para niños y mayores, incremento de los itinerarios seguros, fomento de la calidad medioambiental, mejora del paisaje, etc. De esta manera, en un mundo en el que cualquier figura de la ciudadanía es compleja, intentamos responder a pequeña escala a la cuestión de dónde encontramos espacios que permitan renovar el ejercicio activo de la ciudadanía y sobre qué espacios y procesos pueden fomentar la práctica y el desarrollo de procesos deliberativos que actualicen los vínculos comunitarios. 


\section{Referencias}

Beck, U. y Beck-Gernsheim, E. (2002). Individualization. Londres: Sage.

Lefebvre, H. (1978). El derecho a la ciudad. Barcelona: Ediciones Península.

Moncusí Ferré, A. (2009). Nuevos y viejos vecinos en dos barrios de Valencia (Orriols y Russafa), Departament de Sociologia i Antropologia Social, Ajuntament de València, Àrea de Progrés Humà. Regidoria de Benestar Social i Integració, Valencia.

Sassen, S. (2003). Contrageografías de la globalización. Género y ciudadanía en los circuitos transfronterizos. Madrid: Traficantes de sueños.

SennetT, R. (2012). Juntos. Rituales, placeres y política de cooperación. Barcelona: Anagrama.

Torres, F. (2006). Las dinámicas de la convivencia en un barrio multicultural. El caso de Russafa (Valencia). Papeles del CeIc, vol. 2006/1, papel $\mathrm{n}^{\mathrm{o}} 23$, CEIC (Centro de Estudios sobre la Identidad Colectiva). Bilbao: Universidad del País Vasco.

VvaA (2011). El derecho a la ciudad. Barcelona: Institut dels Drets Humans

VvaA (2009). Metodologías participativas. Madrid: Observatorio Internacional de Ciudadanía y Medio ambiente Sostenible (CIMAS). 
